
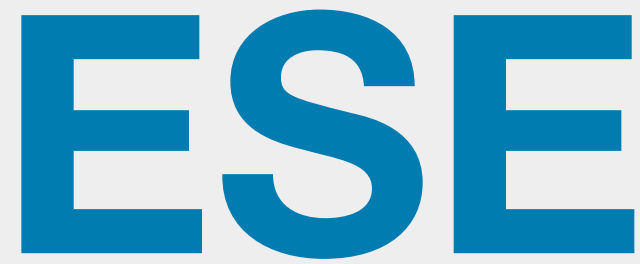

\title{
Emotionale und Soziale
}

Entwicklung in der Pädagogik der Erziehungshilfe und bei Verhaltensstörungen

\section{Heft 3}

Kompetent im NETZwerk:

Realität - Illusion - Vision?!

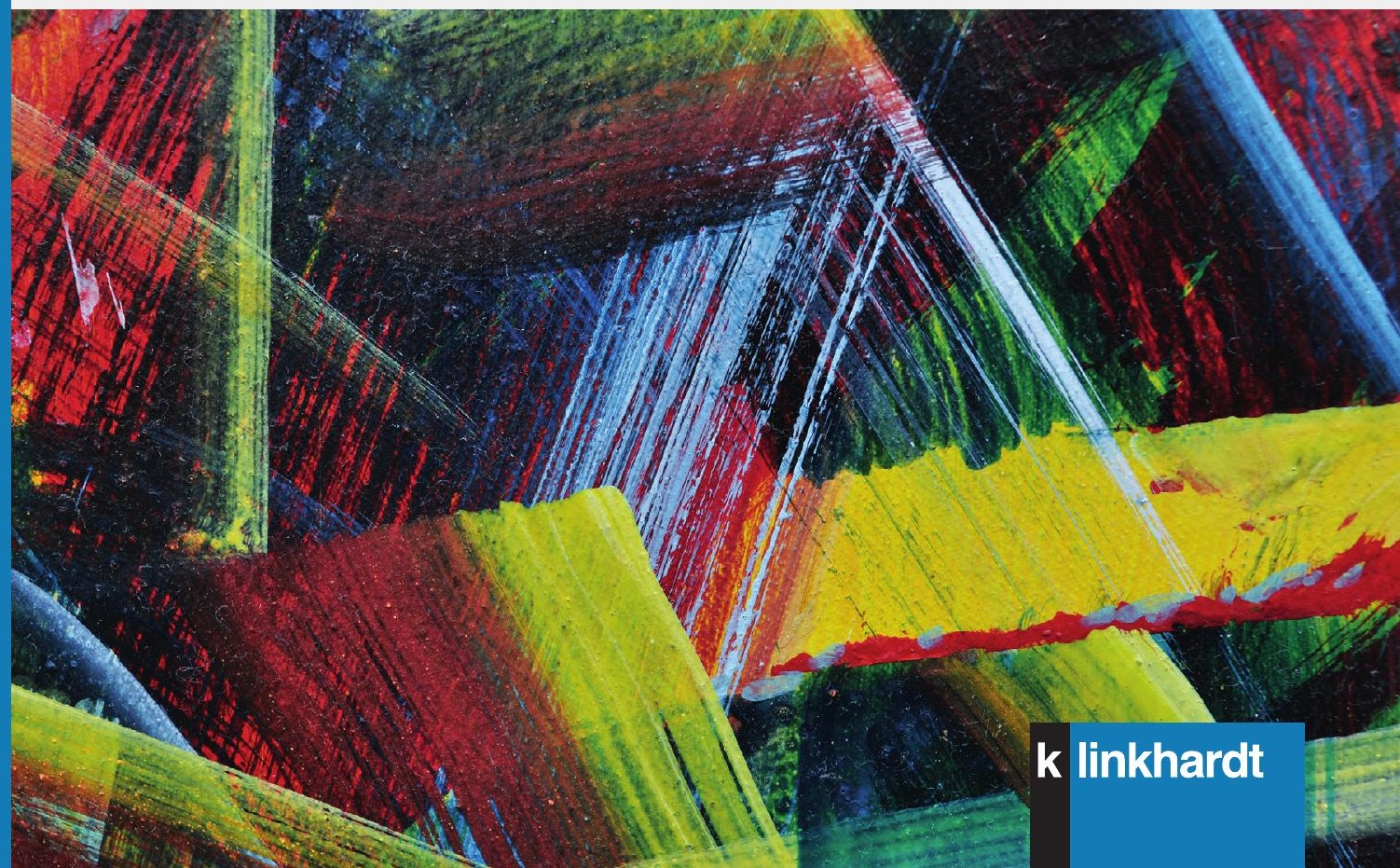




\title{
ESE
}

\author{
Emotionale und Soziale Entwicklung \\ in der Pädagogik der Erziehungshilfe und \\ bei Verhaltensstörungen
}




\section{ESE}

Emotionale und Soziale Entwicklung

in der Pädagogik der Erziehungshilfe und

bei Verhaltensstörungen

\section{Herausgebende Redaktion}

Stephan Gingelmaier (Sprecher der Redaktion)

Werner Bleher

Birgit Herz

Janet Langer

Lars Dietrich

Reinhard Markowetz

\section{Redaktionelle Unterstützung}

Anna Beyer 


\section{ESE}

Emotionale und Soziale Entwicklung in der Pädagogik der Erziehungshilfe und bei Verhaltensstörungen

3. Jahrgang (2021)

Heft 3

Kompetent im NETZwerk: Realität - Illusion - Vision?! 
Die Open Access-Publikation der Zeitschrift ESE wurde von verschiedenen Lehrstüblen und Personen der Dozierendenkonferenz der Forschenden und Lehrenden der „Pädagogik bei Verhaltensstörungen”। des Förderschwerpunkts „emotionale und soziale Entwicklung” finanziert.

Korrespondenzadresse:

Prof. Dr. Stephan Gingelmaier

Psychologie und Diagnostik im Förderschwerpunkt Emotionale und Soziale Entwicklung

PH Ludwigsburg

Reuteallee 46

71634 Ludwigsburg

Erscheinungsweise:

ESE Emotionale und Soziale Entwicklung in der Pädagogik der Erziehungshilfe und bei Verhaltensstörungen erscheint jährlich, jeweils im Sommer.

Die Hefte sind über den Buchhandel zu beziehen.

Das Einzelheft kostet EUR (D) 24,90, im Abonnement EUR (D) 24,90 (ggfs. zzgl. Versandkosten).

Das Abonnement für Studierende kostet EUR (D) 19,90 (ggfs. zzgl. Versandkosten)

nur bei Vorlage einer aktuellen Immatrikulationsbescheinigung.

Bestellungen und Abonnentenbetreuung:

Verlag Julius Klinkhardt

Ramsauer Weg 5

D-83670 Bad Heilbrunn

Tel: +49 (0)8046-9304

Fax: +49 (0)8046-9306

oder nutzen Sie unseren webshop:

www.klinkhardt.de

Bibliografische Information der Deutschen Nationalbibliothek

Die Deutsche Nationalbibliothek verzeichnet diese Publikation in der Deutschen Nationalbibliografie; detaillierte bibliografische Daten sind im Internet abrufbar über http://dnb.d-nb.de.

2021.1. () by Julius Klinkhardt.

Bildnachweis Umschlagseite 1: ( ) Petr Hrbek, 1992, Ohne Titel (Ausschnitt);

mit freundlicher Genehmigung von Ursula Binder und Martina Hoanzl.

Druck und Bindung: AZ Druck und Datentechnik, Kempten.

Printed in Germany 2021.

Gedruckt auf chlorfrei gebleichtem alterungsbeständigem Papier.

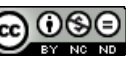

Die Publikation (mit Ausnahme aller Fotos, Grafiken und Abbildungen) ist veröffentlicht unter der Creative Commons-Lizenz: CC BY-NC-ND 4.0 International

https://creativecommons.org/licenses/by-nc-nd/4.0/

ISBN 978-3-7815-5903-5 Digital

doi.org/10.35468/5903

ISBN 978-3-7815-2467-5 Print

ISSN 2629-0170 


\section{Inhaltsverzeichnis}

Editorial der Herausgebenden 7

I Originalia

Perspektiven von Bediensteten des Jugendstrafvollzugs auf pädagogische Beziehung tiefenhermeneutische Einsichten aus einer qualitativ-empirischen Studie Janet Langer, Pierre-Carl Link, Ulrike Fickler-Stang und David Zimmermann

Erfassung der Lehrer*innen-Schüler*innen-Beziehung - ein Überblick über Erhebungsverfahren und -instrumente für die (sonder-)pädagogische Forschung und Praxis

Tatjana Leidig, Tijs Bolz, Émilie Niemeier, Jannik Nitz und Gino Casale

Multiprofessionalität und Netzwerke als Generallösung? -

Ein Plädoyer für einen Perspektivwechsel

Andrea Bethge

Mentalisieren im Netzwerk? Das Adaptive Mentalization-Based Integrative Treatment (AMBIT) als (inter-)professioneller Ansatz im Kontext der Pädagogik bei Verhaltensstörungen Andrea Dlugosch

Professionalität in pädagogischen Zwangskontexten:

Eine Annäherung aus der Perspektive der Pädagogik bei Verhaltensstörungen

Birgit Herz. .78

Professionalisierung im Kontext externalisierender Verhaltensprobleme -

Entwicklung eines Qualifizierungs- und Begleitkonzepts für Lehrkräfte an Förderschulen mit dem Förderschwerpunkt Emotionale und soziale Entwicklung

Tatjana Leidig, Charlotte Hanisch, Ulrike Vögele, Émilie Niemeier, Silke Gerlach

und Thomas Hennemann

Kunstunterricht im Kontext von Unterrichts- und Verhaltensstörungen -

Zur Konzeption eines didaktischen Planungsmodells für Kunst- und

Sonderpädagoginnen und -pädagogen

Daniel Ricci.....

Netzwerk B ${ }^{2}$ : Betrieb und Berufsschule in der inklusiven Ausbildungsvorbereitung eine Fragebogenstudie zu zentralen Bedingungs- und Wirkfaktoren

Francesco Ciociola, Stefanie Roos und Christoph de Oliveira Käppler.

aRT - ein Ansatz zur wissenschaftlich gestützten Schulberatung Janna Rühl, Pascal Schreier, Stephanie Blatz und Roland Stein 
Praxis-Forschungs-Netzwerke am Beispiel des Projekts „Geschwisterklassen“

Désirée Laubenstein und David Scheer

III Praxis und Theorie

Netzwerkarbeit im Kinderschutz. Visionen für die Kooperation bei Gefährdung des Wohls von sonderpädagogisch markierten Kindern

Susanne Leitner.

Umsetzung und Evaluation eines multiprofessionellen und systemübergreifenden Inklusionskonzeptes für Kinder und Jugendliche im Schulalter Karolina Urton, Sophia Hertel und Thomas Hennemann

IV Buchbesprechungen

Das eigensinnige Kind. Über unterdrückten Widerstand und die Formen ungelebten Lebens - ein gesellschaftspolitischer Essay

Pierre-Carl Link

V Forum: Kurzberichte aus den Bundesländern

Bayern: Neuer Standort der Pädagogik bei Verhaltensstörungen in Regensburg

Bernhard Rauh, Pierre-Carl Link und Philipp Abelein

VI Neues aus dem Fach (Zeitraum 2020 - Friuhjahrr 2021) 
Editorial der Herausgebenden 
Editorial zur dritten Ausgabe der Wissenschaftlichen Jahreszeitschrift

Emotionale und Soziale Entwicklung (ESE) in der Pädagogik der Erziehungshilfe und bei Verhaltensstörungen 
Thema:

Kompetent im NETZwerk: Realität - Illusion - Vision?!

\section{Liebe Leserinnen und Leser, liebe Kolleginnen und Kollegen, liebe an unserem Förderschwerpunkt und unserer Fachrichtung Interessierte,}

seit März 2020 beherrscht das Coronavirus weltweit unseren Alltag. In nahezu allen gesellschaftlich relevanten Bereichen spüren wir die Pandemie. Ihre Auswirkungen lähmen und beschäftigen uns alle, in den Mikrosystemen genauso wie auf der Makroebene. Auch die Bundesdozierendenkonferenz im Mai 2020 in München musste schweren Herzens abgesagt werden. An der dritten Ausgabe der Zeitschrift ESE haben wir dennoch festgehalten. Gerade das Tagungsthema „Kompetent im NETZwerk: Realität - Illusion - Vision?!“ sollte nicht unter den Tisch fallen, auch weil sich in den zur Tagung bereits eingereichten Beiträgen Wege aus Krisen und Lösungen für den Umgang unsere fachspezifischen Probleme andeuteten, ohne damals zu wissen, dass Corona die Bewältigung solcher Herausforderungen spürbar verschärfen würde.

Unmissverständlich lehrt uns die Pandemie, dass einzelne Personen gegenüber der Komplexität und Unplanbarkeit von Schieflagen nahezu machtlos sind und nur funktionierende Netzwerke und Verbünde in der Lage sind, mit vereinten Kräften die notwendigen und nachhaltig wirkenden Veränderungen zu erreichen und Antworten auf Fragen zu geben, die sich uns in Theorie und Praxis gleichermaßen aufdrängen.

Mit der ESE III wollten wir ein Forum schaffen, uns als Fachrichtung und unsere manchmal gefühlte Omnipotenz kritisch hinterfragen und Beiträge einfangen, um die Innenansichten über unseren Kreis hinaus der Öffentlichkeit vor-und zur Diskussion zu stellen.

Mit Blick auf unser Fachgebiet sollten seinerzeit im Call for Papers zur Münchener Tagung die folgenden Überlegungen anregen und im Nachgang zu Beiträgen für diese Ausgabe einladen:

Unabhängig davon, ob in quantitativer wie qualitativer Hinsicht Art und Ausprägung von Verhaltensstörungen tatsächlich zunehmen, geben die unterschiedlichsten institutionalisierten Hilfe- und Versorgungssysteme mit ihren professionellen Expertinnen und Experten (z.B. Kinder- und Jugendpsychiatrie, Jugendhilfe, Schulen, Heimeinrichtungen, Gerichtshilfe, Jugendstrafvollzug) vermehrt zu erkennen, dass sie vor immer größeren Herausforde- 
rungen stehen. In der Pädagogik bei Verhaltensstörungen und im Förderschwerpunkt Emotionale und Soziale Entwicklung (ESE) wird hierbei davon ausgegangen, dass die Probleme von Menschen und Systemen mit den unterschiedlichsten Verhaltensstörungen über deren Lebensspanne hinweg von einer Disziplin allein nicht zielführend und nachhaltig bewältigt werden kann, sondern fundamental ein kompetentes Netzwerk und qualitativ hochwertiges, effektives Zusammenarbeiten zwingend einfordern.

Im Rahmen der ESE III fragen wir deshalb, ob wir uns tatsächlich schon kompetent genug im NETZwerk bewegen und wie zielführend und nachhaltig all jene Disziplinen bereits erfolgreich kooperieren, die theoretisch wie berufspraktisch mit hoch belasteten Kindern und Jugendlichen in ihrem Umfeld arbeiten, Lösungen suchen, Bildung, Erziehung und Beziehung in unterschiedlichsten Maßnahmen und Therapien anbieten, aber auch über den gesellschaftlichen Umgang mit den individuellen und sozialen Problemen dieser marginalisierten Gruppe unserer Gesellschaft reflektieren (z.B. aus den Feldern Medizin, Psychologie, Therapie, Schule, Soziale Arbeit, Kriminologie, Philosophie, Soziologie und andere). Auch Grenzen der Kooperation und Kollaboration werden in einzelnen Beiträgen kritisch beleuchtet.

Das Thema „Kompetent im NETZwerk“ lädt ein, aus unterschiedlichsten Perspektiven die Realitäten und Konflikte aufzufalten, Illusionen an- und auszusprechen, aber auch Visionen aufzuzeigen, um das Gelingen der Netzwerkarbeit in den unterschiedlichsten Handlungsund Arbeitsfeldern an den Schnittstellen von Prävention, Intervention, Rehabilitation, Resozialisation, Erziehung, Bildung, Förderung und Therapie für hoch belastete Menschen zwischen gesellschaftlicher Inklusion und Exklusion in die Zukunft zu befördern.

Nun hat die Pandemie die Bedeutung des Themas um ein Vielfaches erhöht, die Auseinandersetzung beschleunigt und auf den Plan gerufen. Schon heute sprechen wir von der "Generation Corona“, wissen um die Breite und Tragweite der Belastungen, können noch nicht abschätzen welche Spätfolgen sich einstellen werden und wie sich die Erfahrungen in den diffus erlebten Coronazeiten auf das Leben jenseits von Schule in allen Lebensbereichen auswirken könnte. Fest steht, dass hoch belastete Kinder und Jugendliche unter Pandemiebedingungen noch stärker leiden und noch schneller an Grenzen kommen. Offene Hilfen und institutionalisierte Förder- und Unterstützungsmaßnahmen kamen und kommen noch immer wieder teilweise vollständig zum Erliegen. Auf Kontaktbeschränkungen folgen nicht nur Beziehungsverluste. Sie schränken viele natürliche Bedürfnisse ein, minimieren den Aufenthalt in sozialen Räumen und verbieten immer wieder auf unbestimmte Zeit die für eine heranwachsende Generation doch so wichtigen soziale Begegnungen für das Zusammenleben und Zusammenhandeln der Menschen. Das soziale und emotionale Erleben unserer Personengruppe wird auf eine harte Probe gestellt. Rückschläge sind vorprogrammiert und Entwicklungen mehr als gefährdet. In den Familien spielen sich auf oft engstem Raum das seelische und körperliche Wohl der Kinder und Jugendlichen gefährdende - Szenen ab, die wiederum zu Beschädigungen bei den Heranwachsenden führen. So verwundert nicht, dass sich Kinder und Jugendliche hochgradig irritiert und allein gelassen fühlen, symptomatisch auf die pandemische Eskalation antworten und in ihrem Verhalten noch auffälliger und herausfordernder werden. Umso mehr braucht es jetzt sehr gut organisierte Netzwerke und ein Mehr an Netzwerkforschung, um diese Gefahren für eine ohnehin schon überlastete, marginalisierte und teilweise schon abgeschriebene Generation abzuwenden. Wenn kein Kind verloren gehen soll, braucht es der Hin- und Zuwendung, zielführende Angebote und insbesondere das Zusammenwirken aller Disziplinen in Netzwerken! Und so schließt sich das Thema Soziales Lernen und Bildung, wie es bei der Bundesdozierendenkonferenz im Juli 2021 
von Susanne Jurkowski und ihrem Team der Universität Erfurt aufgegriffen und in der ESE IV dann abgebildet werden wird, hier nahtlos wie notwendig an.

In der dritten Ausgabe der ESE, die Sie als Leserinnen und Leser nun in den Händen halten, nähern sich über die Rubrik I der Originalia dem Thema Kompetent im NETZwerk zunächst zwei blind begutachtete Beiträge an. Diesen folgen acht Aufsätze in der Rubrik II Tagungsbeiträge und weitere Fachbeiträge, die wie alle weiteren Beiträge eine intensive Begutachtung durch die Herausgebenden über mehrere Feedbackschleifen hinweg durchlaufen haben. Zwei weitere Beiträge aus Praxis und Theorie in Rubrik III schließen daran gut an. Des weiteren finden die Leserinnen und Leser in der Rubrik IV eine Buchbesprechung und in der Rubik V einen Bericht aus den Ländern und deren Studienorten. Zum ersten Mal präsentieren wir in Rubrik VI Neues aus dem Fach - Lesens- und Wissenswertes für den Berichtszeitraum 2020 bis Frühjahr 2021. Damit haben wir die Möglichkeit geschaffen, über Ernennungen, Preise, Berufungen, Promotionen, Habilitationen usw. aus unserer Scientific Community zu informieren. Als Ausdruck unserer Wertschätzung werden wie immer in Rubrik VII alle Kolleginnen und Kollegen genannt, die zum Gelingen dieser dritten Ausgabe der ESE beigetragen haben. Auch und gerade wegen der anhaltend bedrückenden Lage seit März 2020 arbeiten wir an den unterschiedlichsten Standorten wissenschaftlich und in fachpraktischen Projekten, um die Förder- und Unterstützungsmöglichkeiten von hochbelasteten Kindern und Jugendlichen zu verbessern und deren Teilhabe in allen Bereichen der Gesellschaft zu sichern. Als bundesweites Kollegium im deutschsprachigen Raum haben wir nach einer Idee und auf Einladung von Reinhard Markowetz über zwei anregende und kurzweilige Online-Treffen (im November 2020 und März 2021) den Kontakt gehalten. Wir haben uns intensiv ausgetauscht, sind in konstruktive und zielführende Diskussionen eingetreten, so dass alle spüren konnten, wie wichtig es ist, uns selbst als Netzwerk zu verstehen und zu etablieren, um zusammen mit unseren Expertisen nachhaltig wirken zu können.

Deshalb freuen wir uns auch, berichten zu können, dass die Arbeit an unserem noch jungen Vorhaben der ESE-Zeitschrift sehr gut läuft. Wir haben das Gefühl, wahrgenommen zu werden und uns allmählich am pädagogischen Zeitschriftenmarkt etablieren zu können. Möge diese dritte Ausgabe dazu beitragen!

Mit jedem Erscheinen ist die Redaktion erleichtert und dankbar! Auch wenn wir noch den einen oder anderen Fehler machen, lernen wir mit jeder Ausgabe. Natürlich machen wir auch die Erfahrung, dass für die Produktion einer qualitativ hochwertigen Zeitschrift als Ausdruck einer wissenschaftlich intakten wie gesellschaftskritischen Fachgemeinschaft die Einhaltung von Standards unverzichtbar ist. Wenn die Herausgebenden deshalb gelegentlich Beiträge ablehnen oder zur Optimierung einladen, hoffen wir, dass das stets nachvollziehbar ist und transparent bleibt. Gino Casale und Christian Huber (beide Uni Wuppertal) gilt deshalb unser Dank dafür, dass sie sich auf dem Weg zu noch mehr Professionalität aufgemacht haben, um einen wissenschaftlichen Beirat für die ESE zu gewinnen und zu organisieren. Nicht zuletzt danken wir Anna Beyer für die äußerst wertvolle redaktionelle Assistenz und dem gesamten Klinkhardt-Verlag, insbesondere unserem Verleger Andreas Klinkhardt und Thomas Tilsner, von dem wir uns optimal beraten und betreut fühlen, um stets rechtzeitig vor der nächsten Bundesdozierendenkonferenz mit einer druckfrischen Ausgabe aufwarten zu können. 
12 | Editorial der Herausgebenden

Als Herausgeberinnen- und Herausgeber-Team bedanken uns für Ihr Vertrauen, Ihre fachliche Expertise, Ihre (finanzielle) Unterstützung und Ihre Mitarbeit an dieser Ausgabe und freuen uns auf ihre kritisch-konstruktiven Rückmeldungen.

Mit herzlichen und kollegialen Grüßen bis zu einem Wiedersehen in Erfurt aus

Ludwigsburg, Hannover, Rostock, Berlin \& München im April 2021,

Stephan Gingelmaier, Werner Bleher, Birgit Herz, Janet Langer, Lars Dietrich und Reinhard Markowetz

Und bitte denken Sie daran: Die ESE verzichtet bewusst auf eine Kofinanzierung durch Werbung und lebt ausschließlich von Abonnements. Wir laden deshalb Sie, Ihre Kolleginnen und Kollegen, Ihre Bibliotheken, Ihnen bekannte Schulen, Träger und Verbände ein, die ESE zahlreich zu abonnieren! 


\section{ESE}

\section{Emotionale und Soziale Entwicklung in der Pädagogik der Erziehungshilfe und bei Verhaltensstörungen}

\section{Heft 3 \\ Kompetent im NETZwerk: \\ Realität - Illusion - Vision?!}

Die wissenschaftliche Jahreszeitschrift ESE will Inhalte und aktuelle Entwicklungen des Faches Emotionale und Soziale Entwicklung (ESE) in der Pädagogik der Erziehungshilfe und bei Verhaltensstörungen einer breiten Öffentlichkeit zugänglich machen. Aufgrund der hohen gesellschaftlichen Relevanz ihrer Themen dient sie dem kritischen fachlichen Austausch, der Dokumentation der Jahrestagungen und als Informationsplattform der (Bildungs)Politik.

\section{Kompetent im NETZwerk: Realität - Illusion - Vision?!}

Seit März 2020 beherrscht das Coronavirus weltweit unseren Alltag. Die Auswirkungen lähmen und beschäftigen uns alle, in den Mikrosystemen genauso wie auf der Makroebene. Auch die Bundesdozierendenkonferenz im Mai 2020 in München musste schweren Herzens abgesagt werden. An der dritten Ausgabe der Zeitschrift ESE haben wir auch deswegen umso mehr festgehalten. Gerade das Tagungsthema „Kompetent im NETZwerk: Realität - Illusion Vision?!“ kann nicht „ausfallen“, auch weil sich in den zur Tagung bereits eingereichten Beiträgen Wege aus Krisen und Lösungen für den Umgang von fachspezifischen Probleme andeuteten. Unmissverständlich lehrt uns die Pandemie, dass einzelne Personen gegenüber der Komplexität und Unplanbarkeit von Schieflagen nahezu machtlos sind und nur funktionierende Netzwerke in der Lage sind, die notwendigen und nachhaltig wirkenden Veränderungen zu

978-3-7815-2467-5

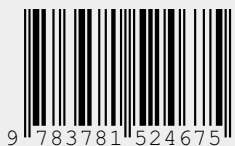

ISSN 2629-0170 erreichen, die sich in Theorie und Praxis aufdrängen. Mit der ESE 3 wollen wir ein Forum schaffen, Beiträge einzufangen, welche die vertretenen hoch relevanten und „vernetzten“ Ansichten über das Fachkollegium hinaus der Öffentlichkeit zur Diskussion stellen. 